Danmarks geologiske Undersøgelse.

IV. Række. Bd. 2. Nr. 3.

\title{
Die Cypraeacea des Daniums von Dänemark und Schonen.
}

\author{
Von
}

F. A. Schilder.

København.

C. A. Reitzel's Forlag. 1928.

Pris $1 \mathrm{Kr}$. 
Danmarks geologiske Undersøgelse.

IV. Række. Bd. 2. Nr. 3.

\title{
Die Cypraeacea des Daniums von Dänemark und Schonen.
}

\author{
Von \\ F. A. Schilder.
}

København.

C. A. Reitzel's Forlag.

1928. 
FR. BAGGES KGL. HOFBOGTRYKKERI KØBENHAVN 


\title{
Die Cypraeacea des Daniums von Dänemark und Schonen. $\left.{ }^{1}\right)$
}

\begin{abstract}
$\mathrm{A}^{\mathrm{u}}$
us dem altbekannten Kalksteinbruche von Faxe (Faxö, Fakse) im südöstlichen Sjælland beschrieb schon 1820 Sснцотнегі²) zwei Steinkerne von Cypraeacea, nämlich $C y$ praeacites bullarius und C. spiratus $\left.{ }^{3}\right)$; hierzu fügte 1902 $\mathrm{RAvN}^{4}$ ) eine dritte Art, Cypraea globuliformis, und ich selbst ${ }^{5}$ ) trennte 1927 Eocypraea danica von bullaria ab. Durch freundliches Entgegenkommen der Herren Museumsinspector J. P. J. Ravn und Prof. K. A. Grönwall wurde mir ermöglicht, im Februar 1928 das reiche Material an Cypraeacea in den Sammlungen des Universitetets Mineralogiske og Geologiske Museum in København (abgekürzt: Køb. Mus.) und der Uni-
\end{abstract}

1) Diese Arbeit ist in den Rahmen einer Reihe von Spezialstudien "Synopsis der Cypraeacea fossiler Lokalfaunen "hineingepasst; der 1. Teil ("Der Kressenberg«) erschien in den Senckenbergiana, 9, 5, p. 196-222 (1927); daselbst sind die hier gebrauchten Abkürzungen der einzelnen Teile der Schale (Sch.) und des Steinkernes (Stk.) ausführlich erklärt: R. = Rücken, B. = Basis, S. = Seiten, AR. bezw. CR. = rechte bezw. linke S., E. = Enden, VE. bezw. HE. = Vorder- bezw. Hinterende, M. = Mündung, AL. bezw. IL. = Aussen- bezw. Innenlippe, AZ. bezw. IZ. = die dort befindlichen Zahnfalten (Z.), TZ. = vorderster IZ., Sp. = Spira.

$\left.{ }^{2}\right)$ Schlotheim (1820) Petrefaktenkunde, p. 117.

$\left.{ }^{3}\right)$ Porcellanites seelandicus Schlotheim (1813) Leonhard's Taschenb. Miner., 7, p. 103 von ebenda ist ein noch älterer Name für ein Fossil von Faxe, aber nur ein nomen nudum; nach BronN (1848) Index palaeont., p. 384 soll er mit Cypraea spirata ident sein.

4) J. P. J. Ravn (1902) Kong. Danske Vidensk. Selsk. Skr., Nat. Mat. Afd., (6) 11, p. 225.

$\left.{ }^{5}\right)$ F. A. Schilder (1927) Arch. Naturgesch., 91, 10, p. 74, 129. 
versitets Geologisk-Mineralogiska Institution in Lund (abgekürzt: Lund Mus.) zu studieren ${ }^{1}$ ). Den beiden genannten Herren fühle ich mich für ihre stete Hilfsbereitschaft zu grossem Danke verpflichtet, ebenso dem Herrn S. A. Kärfve, Disponent bei der Skånska Cement Aktiebolaget, der mir nach ungewöhnlich gastfreiem Empfange die Besichtigung des grossen Kalkbruches von Limhamn bei Malmö gestattet hat; mit Erlaubnis des Bruchmeisters Jensen konnte ich auch den Kalkbruch von Faxe in Augenschein nehmen.

Die Untersuchung der Cypraeacea führte zur Unterscheidung von mindestens 10 Formen von folgenden 5 Fundorten:

Fx $=$ Faxe (Sjælland): Korallen- und Bryozoenkalk $\left.{ }^{2}\right)$ mit Ausnahme des Fundortes FM. (Køb. Mus.: zusammen 500 Stücke $\left.^{3}\right)$ ).

FM = Faxe-Mitte: darunter verstehe ich die in kurzer Zeit wohl gänzlich abgebaute, im Volksmunde »Ravns Nase« genannte Partie in der Mitte des der Bahnstation Stubberup nächstgelegenen Teiles des Faxe-Bruches; sie besteht aus auffällig feinem weissem Kalk und enthält die Fossile in vorzüglichem Erhaltungszustande, die Mollusken meist mit erhaltener Schale ${ }^{4}$ ). (Køb. Mus., einige Stücke auch in coll. Jensen-Faxe ${ }^{5}$ ), zusammen 39 Stücke).

1) Ich war von der grossem Zahl der in beiden Museen vorhandenen Individuen sehr angenehm überrascht, umso mehr, da in den anderen Museen des Kontinentes nur selten höchstens 1-2 Steinkerne von Faxe zu finden sind.

$\left.{ }^{2}\right)$ Die Fossile des Korallen- und Bryozoenkalkes waren im Køb. Mus. leider nicht mehr zu trennen.

$\left.{ }^{3}\right)$ Die angegebenen Stückzahlen beziehen sich nur auf die in messbarem Zustande erhaltenen Individuen; die mir freundlicherweise überlassenen Desiderata sind miteinbegriffen.

$\left.{ }^{4}\right)$ Nach brieflicher Mitteilung des Herrn Dr. H. ØDum ist dieser Teil geologisch jünger als die übrigen Partien des FaxeBruches und wahrscheinlich gleich alt mit Limhamn.

${ }^{5}$ ) In coll. Jensen u. a. der grösste Stk. von Cypraedia separabilis maxima und die erhaltene Sch. von Eocypraea bullaria. 
St $=$ Stevns Klint (Sjælland): Bryozoenkalk $\left.{ }^{1}\right) .($ Køb. Mus. : 1 Stück).

$\mathrm{Ag}=$ Aggersborg Gaard (Jylland): Bryozoenkalk²). (Køb. Mus.: 32 Stück).

Lim = Limhamn (früher auch als Annetorp bezeichnet) bei Malmö (Skåne): weisser Kalk. (Lund Mus. und Køb. Mus. ${ }^{3}$ ): zusammen 208 Stücke).

\begin{tabular}{|c|c|c|c|c|c|}
\hline \multirow{2}{*}{$\begin{array}{l}\text { Familie und Unterfamilie } \\
\text { Genus, Species und Subspecies }\end{array}$} & \multicolumn{5}{|c|}{ Zahl der untersuchten Stücke } \\
\hline & $\mathrm{Fx}$ & FM & St & Ag & Lim \\
\hline \multirow{2}{*}{\multicolumn{6}{|c|}{$\begin{array}{l}\text { Amphiperasidae: Eocypraeinae } \\
\text { Cupraedia separabilis nov. }\end{array}$}} \\
\hline & 23 & 1 & - & - & 16 \\
\hline - $\quad$ - minuta nov. & - & - & - & 2 & 一 \\
\hline maxima nov. & - & 3 & - & 一 & 4 \\
\hline Ravni nov........... & 1 & - & - & - & - \\
\hline Eocypraea danica Schild......... & 41 & 11 & - & 1 & 36 \\
\hline - bullaria Schloth....... & 110 & 3 & - & 6 & 25 \\
\hline \multicolumn{6}{|l|}{ Cypraeidae: Gisortiinae } \\
\hline Vicetia faxensis nov...... & 1 & - & 一 & - & - \\
\hline \multicolumn{6}{|l|}{ Cypraeidae: Cypraeinae } \\
\hline Palaeocypraea spirata Schloth.... & 274 & 21 & 1 & 23 & 60 \\
\hline - $\quad$ suecica nov...... & 34 & - & - & - & 67 \\
\hline Protocypraea globuliformis Ravn. & 16 & - & - & - & - \\
\hline
\end{tabular}

Das Studium der Steinkerne lässt zwar die Gattungszugehörigkeit meist sicher erkennen, reicht aber nicht aus, die einzelnen Arten auseinanderzuhalten; allerdings kann mittels variationsstatistischer Methoden meist ein deutlicher Hinweis auf eine weitere Teilbarkeit von »Arten«, die bisher für einheitlich gehalten wurden, gewonnen werden. Wegen

1) Vergleiche K. A. Grönwall (1899), Geol. För. Stockh. Förh., 21, p. 371.

$\left.{ }^{2}\right)$ Vergleiche H. Øрum (1926): Studier over Daniet i Jylland og paa Fyn.

$\left.{ }^{3}\right)$ Im Køb. Mus. befinden sich nur 39 Stücke von Limhamn (1 Cypraedia separabilis, 1 Eocypraea bullaria, 3 E. danica, 12 Palaeocypraea spirata und $22 P$. suecica), alle übrigen im Lund Mus. 
3. 6

der durch den vielfach ungenügenden Erhaltungszustand der untersuchten Steinkerne bedingten Unsicherheit der Messungen habe ich mich in dieser Studie darauf beschränkt, nur die sicher trennbaren Formen unter eigenen Namen zu beschreiben, obgleich ich für wahrscheinlich halte, dass künftige Forschungen an besser erhaltenem Materiale von vielleicht neuen Fundplätzen folgende weitere Artteilung gestatten werden:

Cypraedia separabilis (s. str.): eine kleinere und eine grössere Form (Typus).

Eocypraea danica: eine meist kleinere Form von Fx und eine meist grössere von FM (Typus) und Lim, von FM überdies vielleicht eine mehr längliche und eine kugelige Form (Typus).

Eocypraea bullaria: eine kleinere (Typus) und eine grössere Form.

Palaeocypraea spirata: eine grössere Form von Fx (Typus) und eine unmerklich kleinere von FM, Ag und Lim; ausserdem in Fx eine kugelige Abart und in $\mathrm{Ag}$ eine gestreckte Form (= suecica?).

Palaeocypraea suecica: eine kleine, mittlere und grosse (Typus) Form ohne Beziehung zum Fundort.

Bestimmungstabelle der Schalen (soweit bekannt).

la Sp. involut, von aussen niemals sichtbar, M. hinten gebogen, HE. nicht, VE. wenig vorgezogen, Z. reichen nicht bis zur HE.-Spitze.

$2 \mathrm{a}$..Sch. meist 12-18 mm lang, VE. allmählich verschmälert und stärker vorgezogen .. Eocypraea danica.

$2 \mathrm{~b}$..Sch. meist 15-32 mm lang, VE. mehr plötzlich verschmälert und weniger stark vorgezogen....... .................... Eocypraea bullaria.

lb Sp. vorgezogen, unter dem Callus meist erkennbar, M. hinten gerader, HE. und VE. sehr stark vorgezogen, oft etwas rückgebogen, $Z$. reichen bis zur HE.-Spitze, Sch. 13-21 mm lang........... ................... Palaeocypraea spirata. 
Bestimmungstabelle der Steinkerne.

la Sp. involut, d. h. die stets schmale Spitze tritt höchstens kurz aus der Umfassung des letzten Umganges hervor, der sie besonders auf der linken Seite eng umschliesst.

2a . Das vom letzten Umgange gebildete Sp.-Loch ist rund, die Sp.-Spitze liegt vertieft in der Mitte, der letzte Umgang am Sp.-Loch dick, gleichmässig hineingerundet.

3a .... Stk. kugelig, R. und S. gleichmässig gerundet.

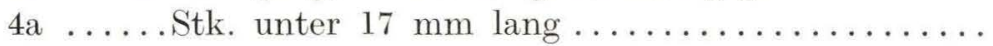

............ Cypraedia separabilis minuta.

4b ..... Stk. 18-32 mm lang. Cypraedia separabitis s. str.

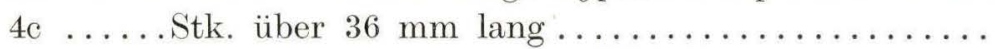

........... Cypraedia separabilis maxima.

$3 b$....Stk. elliptisch, R. mehr gedrückt, S. mehr gerade.. .................... Cypraedia Ravni.

$2 b$. Das vom letzten Umgange gebildete Sp.-Loch ist nach rechts oben erweitert, die Sp.-Spitze liegt am linken Rande, der letzte Umgang am Sp.-Loch dünn und scharfkantig.

5a ....Stk. meist 9-15 mm lang, bauchig, S. gleichmässig gerundet ............. Eocypraea danica.

$5 b$... Stk. meist $13-27 \mathrm{~mm}$ lang, mehr gedrückt, S. (bes. AR.) mehr gerade und vorn mehr plötzlich verschmälert............ Eocypraea bullaria.

1b Sp. nicht involut, d. h. breit, freiliegend, vom relativ schmaleren letzten Umgange links nicht überdeckt, eher rechts vom umgebogenen HE. der AL.

6 a ..Stk. über $50 \mathrm{~mm}$ lang, gestreckt zylindrisch........ ................. Vicetia faxensis.

$6 \mathrm{~b}$..Stk. stets unter $40 \mathrm{~mm}$ lang, kugelig, eiförmig oder kurz zylindrisch.

7a ....Sp. (alle Umgänge einschliesslich des Protoconch) stark vorgezogen.

8a ......Stk. 6-17 mm lang, AR. ziemlich gerade und erst vorn plötzlich umgebogen. Palaeocypraea spirata. 
3. 8

8b ..... Stk. 17-32 mm lang, AR. von $2 / 3$ der Länge an nach vorn zu gleichmässig und allmählich gebogen.............. Palaeocypraea suecica.

$7 b$...Sp. sehr wenig vorspringend, die Umgänge fast in einer Ebene liegend.. Protocypraea globuliformis.

Die Mehrzahl der 780 Stücke ist so gut erhalten, dass ihre grösste Länge ${ }^{1}$ ), Breite und Höhe auf ${ }^{1 / 10} \mathrm{~mm}$ genau gemessen und aus diesen Maassen der Breitenindex $=100 \times$ Breite: Länge und der Höhenindex $=100 \times$ Höhe: Breite berechnet werden konnten; bei den übrigen Stücken, die nur in 2 oder gar nur in 1 Dimension sicher messbar sind, wurden die fehlenden Maasse nach den häufigsten vorkommenden mittleren Verhältniszahlen der 3 Dimensionen ergänzt. Die Ergebnisse dieser Messungen sind in der nachstehenden Tabelle zusammengestellt, und zwar bedeuten die 3 Ziffern in jeder Kolonne das festgestellte Minimum, Mittel und Maximum.

\begin{tabular}{|c|c|c|c|c|c|c|}
\hline & & $\begin{array}{c}\text { Länge } \\
\text { mm }\end{array}$ & $\begin{array}{l}100 \times \\
\text { Breite } \\
\text { Länge }\end{array}$ & $\begin{array}{l}100 \times \\
\text { Höhe } \\
\text { Breite }\end{array}$ & $\begin{array}{l}100 \times \\
\text { Spirabreite } \\
\text { Stk.-Breite }\end{array}$ & $\begin{array}{l}\text { Stk.-Länge } \\
=\% \text { der } \\
\text { Sch.-Länge }\end{array}$ \\
\hline Cypraedia separabilis & Stk. & $18-24-32$ & $78-85-93$ & $80-83-86$ & $12-15-16$ & $?$ \\
\hline minuta $\ldots$ & Stk. & $14-15-16$ & 84 & 85 & $?$ & $?$ \\
\hline $\operatorname{maxima} .$. & Stk. & $36-38-42$ & $76-83-90$ & $81-83-85$ & 16 & $?$ \\
\hline Ravni.... & Stk. & 26 & 74 & 84 & 14 & $?$ \\
\hline Eocypraea danica.... & Sch. & $12-15-18$ & $66-74-76$ & $80-85-89$ & 一 & 一 \\
\hline$-\ldots$ & Stk. & $6-12-18$ & $77-85-95$ & $80-84-87$ & $21-22-24$ & 80 \\
\hline bullaria... & Stk. & $10-19-27$ & $73-80-88$ & $76-82-87$ & $17-20-24$ & 85 \\
\hline Vicetia faxensis ..... & Stk. & 54 & 58 & 77 & 44 & $?$ \\
\hline Palaeocypraea spirata & Sch. & $13-16-21$ & $59-63-67$ & $79-84-88$ & - & 一 \\
\hline- & Stk. & $6-12-17$ & $74-83-92$ & $75-81-87$ & $28-36-42$ & 69 \\
\hline suecica & Stk. & $17-24-32$ & $69-78-84$ & $78-82-86$ & $41-46-51$ & $?$ \\
\hline $\begin{array}{l}\text { Protocypraea globuli- } \\
\quad \text { formis ............ }\end{array}$ & Stk. & $10-13-14$ & $83-91-96$ & $83-87-92$ & $38-40-42$ & $?$ \\
\hline
\end{tabular}

1) Um die genaue Länge einer möglichst grossen Zahl von Steinkernen sicher bestimmen zu können, erfolgte ihre Messung bei den Arten der beiden Cypraeacea-Familien in verschiedener Weise: den einen Endpunkt der gemessenen Strecke bildete stets das Vorderende des Rückens ausschliesslich der Ausfüllung des vorderen Ausgusses der Mündung, den anderen bei den Amphi- 
Genus Cypraedia Swainson.

Der dicke, zum engen Sp.-Loch gleichmässig eingerollte Hinterrand des letzten Umganges der beiden Arten aus dem Danium, separabilis und Ravni, stimmt mit Cypraedia bavarica Schild. vollkommen überein; die durch die häufige Sichtbarkeit der längsverlaufenden Anwachsstreifen gekennzeichnete Dünne der Schale sowie die bei Ravni erhaltene Gitterskulptur (nicht bloss dichte Spiralrippung, die ja auch bei Eocypraea an jungen Schalen bezw. auf den inneren Umgängen adulter Schalen vorkommt!) bestätigen die Zugehörigkeit zu Cypraedia. Nach ihrer Gestalt und Skulptur gehören beide Arten zur Gruppe von elegans Sow., aber nur die Rasse minuta kommt dieser auch in der Grösse gleich, die anderen Formen sind merklich grösser als die meisten Cypraedia des Tertiär; separabitis scheint in Grösse und Gestalt der mitteleocänen bavarica separata vom Kressenberg sehr nahe zu stehen.

\section{Cypraedia separabilis nov. sp. $=$ ? Cypraeacites bullarius Schlotheim (1820) Petrefakt., p. 117 (pars).}

Steinkern: Kugelig, bauchig, undeutlich längsgestreift ${ }^{1}$ ) mit gleichmässig gerundetem R., nur wenig vorgezogenem VE. und gleichmässig gebogenen S.; M. hinten stark gebogen, AL. wulstig'2), VE. der IL. gerade, Sp. schmal, kurz, meist ganz in engem Loch mit ringsum dick gerundeten Wänden versinkend. $28 \times 23 \times 19 \mathrm{~mm}$ (Typus von $\mathrm{Fx}, \mathrm{K} ø b$. Mus., fig. 6).

Die Schale hatte nach Abdrücken am Typus der subsp. maxima (siehe unten!) trotz ihrer Grösse äusserst feine und

perasidae das Hinterende der Aussenlippe, bei den Cypraeidae der Hinterrand des letzten Umganges in der Mitte des Rückens, also unter Ausschluss der Spira, da deren das Hinterende der Aussenlippe überragende Spitze oft abgebrochen ist.

1) Am deutlichsten bei 1 separabilis s. str. (26 mm) und 2 maxima $(38 \mathrm{~mm})$ von FM sowie 1 minuta $(16 \mathrm{~mm})$ von Ag.

$\left.{ }^{2}\right)$ Die AL. ist aber meist weniger gekielt als bei Eocypraea bullaria, ein Merkmal, das auch die Stellung der Art zu Cypraedia bestätigt. 
3. 10

dichte AZ. (vergleichbar etwa Globulina utriculata Lam.); 1 Stk. $(22 \times 20 \times 17 \mathrm{~mm}$ von Lim, Lund Mus.) zeigt auf der IL. und am CR. etwa 13 Querrippen, offenbar Reste einer Gitterskulptur (die feineren Zwischenrippen und die Längsrippung sind nicht mehr erkennbar).

Abänderungen. Die Variabilität in der Länge der Stk. ist sehr gross:

\begin{tabular}{|c|c|c|c|c|c|c|}
\hline \multirow{2}{*}{$\mathrm{mm}$} & \multicolumn{6}{|c|}{ Ort } \\
\hline & $\mathrm{Fx}$ & FM & $\mathrm{Ag}$ & $\operatorname{Lim}$ & Summe & oder $^{1}$ ) \\
\hline 14 & - & - & 1 & - & 1 & 1 \\
\hline 15 & - & - & - & - & - & 1 \\
\hline 16 & - & - & 1 & - & 1 & 1 \\
\hline 17 & - & - & - & - & - & \\
\hline 18 & - & - & - & 2 & 2 & 2 \\
\hline 19 & 1 & - & - & - & 1 & \\
\hline 20 & 4 & - & - & 2 & 6 & 7 \\
\hline 21 & - & - & - & 3 & 3 & \\
\hline 22 & 4 & - & - & 1 & 5 & 8 \\
\hline 23 & 1 & - & - & 1 & 2 & 6 \\
\hline 24 & 3 & - & - & 1 & 4 & 6 \\
\hline 25 & 1 & - & - & - & 1 & 2 \\
\hline 26 & - & 1 & - & 1 & 2 & $J$ \\
\hline 27 & 1 & - & - & 1 & 2 & 5 \\
\hline 28 & 2 & - & - & 1 & 3 & 5 \\
\hline 29 & 2 & - & - & 2 & 4 & \\
\hline 30 & 3 & - & - & - & 3 & 7 \\
\hline 31 & 1 & - & - & - & 1 & \\
\hline 32 & - & - & - & 1 & 1 & 2 \\
\hline 33 & - & - & 一 & - & 一 & \\
\hline 34 & - & - & - & - & - & \\
\hline 35 & - & - & - & - & - & \\
\hline 36 & - & - & - & 1 & 1 & 1 \\
\hline 37 & - & - & - & - & - & \\
\hline 38 & - & 2 & - & 2 & 4 & 4 \\
\hline 39 & - & - & - & 1 & 1 & 1 \\
\hline 40 & - & - & - & - & - & 1 \\
\hline 41 & - & 一 & - & 一 & - & 1 \\
\hline 42 & - & 1 & - & - & 1 & 1 \\
\hline
\end{tabular}

1) Zur besseren Uebersichtlichkeit wurden hier die Individuen von je 2 Grössenklassen zusammengezogen. 
Aus dieser Tabelle ist zu erkennen, dass man 3 auch in ihrer Verbreitung verschiedene Formen unterscheiden kann:

\begin{tabular}{c|c|c}
\hline Name & Ort & Länge \\
\hline subsp. minuta nov. $\left.{ }^{1}\right) \ldots \ldots \ldots$ & Ag & $14-15-16$ \\
separabilis s. str. .......... & Fx, (FM), Lim & $18-24-32$ \\
subsp. maxima nov. $\left.{ }^{2}\right) \ldots \ldots \ldots$ & FM, Lim & $36-38-42$ \\
\hline
\end{tabular}

Die Verteilung der Individuen auf die einzelnen Grössenklassen lässt ferner vermuten, dass die Form separabilis s. str. selbst noch weiter geteilt werden könnte, nämlich in eine kleinere $(17-25 \mathrm{~mm})$ und eine grössere, typische Form (26$32 \mathrm{~mm}$ ), da mittelgrosse Stücke (etwa $25 \mathrm{~mm}$ lang) weit seltener sind als kleine $(22 \mathrm{~mm})$ oder grosse $(29 \mathrm{~mm})$; diese beiden Formen kommen jedoch in Fx und Lim in etwa gleicher relativer Häufigkeit nebeneinander vor, weshalb ich von ihrer nomenklatorischen Trennung vorläufig Abstand nehme.

\section{Cypraedia Ravni nov. sp.}

= Cypraea bullaria Ravn (1902) Kong. Danske Vidensk. Selsk. Skr., Nat. Mat. Afd., (6) 11, tab. 2, fig. 5 (nec fig. 4, nec Schlotheim).

Steinkern subzylindrisch, R. gedrückt, etwas abgeflacht, mit sehr dichten Längsstreifen und undeutlichen Querrippen (etwa 8 längs der AL.), S. fast parallel, nach vorn erst wenig, dann plötzlich stark gebogen; am HE. der IL. ist noch die für Cypraedia typische Gitterskulptur vorzüglich erhalten: die Querrippen sind ebenso dicht wie die Längsrippen und unter-

1) Als Typus bezeichne ich das $16 \times 13 \times 11 \mathrm{~mm}$ grosse Stück von Ag, das im April 1898 von GröNwall gesammelt wurde (Køb. Mus.); es zeigt die typische Längsstreifung am VE. sehr deutlich.

2) Als Typus bezeichne ich das $38 \times 32 \times 27 \mathrm{~mm}$ grosse Stück von FM (Køb. Mus.) mit deutlich erkennbarer Längsstreifung und Abdrücken der Bezahnung. 
einander alle gleichmässig stark. 25 1/2 × $19 \times 16 \mathrm{~mm}$ (Typus von Fx, Køb. Mus., fig. 7).

Die von Ravr gegebene Photographie zeigt die Gestalt und die Längsstreifung des $R$. vorzüglich, doch wird die wohlerhaltene dichte Gitterskulptur der nicht abgebildeten Unterseite nirgens erwähnt; das Unikum zeigt wie separabilis Abdrücke von äusserst feinen $\mathrm{AZ}$.

Obwohl nur in 1 Stück bekannt, ist Ravni durch die gänzlich abweichende Gestalt des Stk. von separabilis sicher zu trennen.

\section{Genus Eocypraea Cossmann.}

Die gut erhaltenen Schalen der beiden Arten danica und bullaria lassen typische Eocypraea erkennen, die sich durch nichts Wesentliches von den übrigen Arten (Albian bis OberEocän) unterscheiden; am Steinkern weichen die Merkmale der Sp. von denen späterer Eocypraea (wie inflata Lam. vom Kressenberg) merklich ab und der dorso-labrale Ausschnitt des letzten Umganges deutet auf ihre Verwandtschaft mit den eocänen Sphaerocypraea hin, doch fehlt bei danica und bullaria noch stets der für Sphaerocypraea charakteristische Vorsprung am dorso-columellaren Rande des Sp.-Loches. Auch das obgleich nur ausnahmsweise vorkommende stärkere Vorspringen der Sp.-Spitze ist ein Zeichen für eine relativ primitive Entwicklungsstufe der beiden Arten des Danium.

\section{Eocypraea danica Schilder.}

= Cypraea bullaria Ravn (1902) Kong. Danske Vidensk. Selsk. Skr., Nat. Mat. Afd., (6) 11, tab. 2, fig. 4 a-c (nec fig. 5, nec Schlotheim).

= Eocypraea danica Schilder (1927) Arch. Naturgesch., 91, 10, p. 74, 129.

Schale: Fast kugelig, VE. etwas, HE. kaum vorgezogen, S. gerundet, nur an den E. undeutlich schmal gerandet, M. hinten leicht gebogen, vorn etwas erweitert, VE. der AL. steil decliv, AZ. tiefliegend, kurz faltenförmig, in der Mitte 
auf die Stirnseite der AL. beschränkt, TZ. faltenförmig, randständig, meist undeutlich, gefolgt von einer schrägen Falte (= Zwischen-Z.), nur durch ganz unauffällige Lücke getrennt von den IZ., die vorn etwas schräg und kurzfaltig, in der Mitte und hinten quergestellt, fein, dicht und kurz sind; Fossula sehr breit, aber seicht, glatt, mit einem schwachen Eindruck am glatten Innenrande. $14 \frac{1}{2} \times 10 \frac{1}{2} \times 9 \mathrm{~mm}$ mit $20 \mathrm{AZ}$., 1 TZ. und 15 IZ. (Typus von Fx, Køb. Mus., von Ravn abgebildet).

Steinkern: Kugelig, mit gleichmässig gerundeten S. und nur wenig vorgezogenem VE., M. hinten wenig gebogen, AL. dick, etwas kielartig, Sp. ziemlich klein, knopfförmig im linken Teile des nach rechts meist deutlich erweiterten Ausschnittes des letzten Umganges gelegen, der das Sp.-Loch scharfkantig umgrenzt. $11 \times 9 \frac{1}{2} \times 8 \frac{1}{2} \mathrm{~mm}$ (Exemplar von Fx, Køb. Mus., fig. 11).

Abänderungen. Die Steinkernlänge $(=80 \%$ der Schalenlänge) der untersuchten Stücke beträgt:

\begin{tabular}{r|r|r|r|r|r|r}
\hline \multirow{2}{*}{$\mathrm{mm}$} & \multicolumn{6}{|c}{ Ort } \\
\cline { 2 - 7 } & Fx & FM & Ag & Lim & Summe & oder \\
\hline 6 & 2 & - & - & - & 2 & 2 \\
7 & 1 & - & - & - & 1 & 2 \\
8 & 1 & - & - & - & 1 & \\
9 & 6 & - & - & 3 & 9 & 24 \\
10 & 12 & 1 & 1 & 1 & 15 & \\
11 & 10 & 3 & - & 5 & 18 & 35 \\
12 & 8 & 3 & - & 6 & 17 & \\
13 & - & 1 & - & 10 & 11 & 20 \\
14 & 1 & 2 & - & 6 & 9 & \\
15 & - & - & - & 3 & 3 & 3 \\
16 & - & - & - & - & - & \\
17 & - & - & - & 1 & 1 & 3 \\
18 & - & 1 & - & 1 & 2 & \\
\hline Mittel in & 10.3 & 12.5 & $?$ & 12.7 & 11.5 & \\
mm & & & & & & \\
\hline
\end{tabular}

Die Stücke von Fx sind alsio durchschnittlich etwas kleiner als die von FM und Lim; die Variationskurven aller Fundorte 


\section{14}

für sich scheinen aber eingipfelig zu sein, höchstens dass in FM und Lim eine grössere Form abgetrennt werden könnte.

In der Gestalt der Schale scheinen 2 Varietäten vorzukommen: die typische kurze, plumpe, hochgewölbte Form (fig. 2 von FM. coll. aut.) hat das VE. der AL. steiler decliv und die AZ. tiefer liegen als die etwas mehr gestreckte Form (fig. 1 von FM, Køb. Mus.).

Die Zahl der Z. des Typus ist auffällig gering; sonst beträgt sie nach 8 untersuchten Stücken bei der

Sch.-Länge 13-14 mm = Stk.-Länge 10-11 mm meist $23 \mathrm{AZ}$. und $18 \mathrm{IZ}$.,

Sch.-Länge $15-16 \mathrm{~mm}=$ Stk.-Länge $12-13 \mathrm{~mm}$ meist $24 \mathrm{AZ}$. und $19 \mathrm{IZ}$.

Sch.-Länge $17-18 \mathrm{~mm}=$ Stk.-Länge $14-15 \mathrm{~mm}$ meist $25 \mathrm{AZ}$. und $20 \mathrm{IZ}$.

ohne den TZ. gerechnet.

\section{Eocypraea bullaria Schlotheim.}

= Cypraeacites bullarius Schlotheim (1820) Petrefakt., p. $\left.117(\text { pars })^{1}\right)$.

$=$ Cypraea bullaria Lyell (1840) Trans. Geol. Soc. London, (2) 5, p. 249, tab. 18, fig. 1-3.

Schale ähnlich danica, aber grösser, das VE. weniger vorgezogen, mehr plötzlich verschmälert, daher der CR. im

1) Nach Schlotheim's Angabe "bis fast 2 Zoll" ist nicht $\mathrm{zu}$ zweifeln, dass sich unter seinen 10 bullaria auch wenigstens 1 Cypraedia separabilis maxima befand, nach dem relativ seltenen Vorkommen dieser Form in Faxe ist aber nicht anzunehmen, dass alle seine Stücke zu Cypraedia gehörten. Da die Beschreibung auf die Stk. sämtlicher Cypraedia und Eocypraea passt und Schlotнeim keinen »Typus bezeichnet bezw. abgebildet hat, ist für die Zuteilung des Namens bullaria zu einer der vermengten Arten der nächste revidierende Autor massgebend: die Abbildung Lyell's lässt einen $251 / 2 \mathrm{~mm}$ langen Stk. mit ziemlich parallelen S. und nach rechts erweitertem Sp.-Loch erkennen, also unzweideutig die von mir unter bullaria verstandene Form; die Abbildungen Ravv's müssen demgegenüber aus Prioritätsgründen unberücksichtigt bleiben. 
vorderen Drittel stärker ausgebogen und vorn jäher zugespitzt, die vorderen IZ. mehr gedrängt; wahrscheinlich war der AR. auch etwas deutlicher gerandet. $31 \frac{1}{2} \times 23 \times 21 \mathrm{~mm}$ (Exemplar von FM, coll. JENSEN) und einige Abdrücke von ähnlichen Dimensionen (von Fx, Køb. Mus.).

Steinkern im Vergleiche zu danica grösser, mehr gedrückt, die S. (besonders der AR.) mehr gerade und beiderends jäher verschmälert, der äusserste Umgang rechts oberhalb der Sp. meist noch mehr ausgeschnitten. $15 \times 12 \times 10 \mathrm{~mm}$ (Neotypus ${ }^{1}$ )) von Fx, Køb. Mus., fig. 8).

Abänderungen. Die Variabilität in der Grösse der Stk. ist hier wiederum gross:

\begin{tabular}{l|r|r|r|r|r|r}
\hline \multirow{2}{*}{$\mathrm{mm}$} & \multicolumn{7}{|c|}{ Ort } \\
\cline { 2 - 6 } & Fx & FM & Ag & Lim & Summe & oder \\
\hline 10 & 1 & - & - & - & 1 & 1 \\
11 & - & - & - & - & - & 1 \\
12 & 1 & - & - & - & 1 & \\
13 & 7 & - & 2 & - & 9 & 20 \\
14 & 6 & - & 4 & 1 & 11 & \\
15 & 11 & 1 & - & - & 12 & 19 \\
16 & 6 & - & - & 1 & 7 & \\
17 & 13 & 1 & - & 4 & 18 & 32 \\
18 & 6 & - & - & 8 & 14 & \\
19 & 14 & - & - & 5 & 19 & 28 \\
20 & 6 & - & - & 3 & 9 & \\
21 & 5 & - & - & 1 & 6 & 16 \\
22 & 8 & - & - & 2 & 10 & \\
23 & 11 & - & - & - & 11 & 17 \\
24 & 6 & - & - & - & 6 & \\
25 & 2 & - & - & - & 2 & 5 \\
26 & 3 & - & - & - & 3 & \\
27 & 4 & 1 & - & - & 5 & 5 \\
\hline
\end{tabular}

Es scheint, dass auch bei bullaria eine kleinere Form (10$20 \mathrm{~mm}$, Mittel $\left.=16 \frac{1}{2} \mathrm{~mm}\right)$ und eine grössere Form $(21-$

1) Bis zur Auffindung der Original-Exemplare Schlotheim's und zur Bezeichnung eines Lectotypus aus denselben habe ich einen gut erhaltenen Stk. im Køb. Mus. als Neotypus ausgewählt. 
$27 \mathrm{~mm}$, Mittel $=23 \frac{1}{2} \mathrm{~mm}$ ) unterschieden werden könnte; die grössere Form kommt jedoch mit Ausnahme von Ag (und Lim ?) an allen Fundorten vor, allerdings in Fx am häufigsten. Die mittlere Grösse der kleineren Form beträgt in Ag $13 \frac{1}{2} \mathrm{~mm}$, in Fx $15 \frac{1}{2} \mathrm{~mm}$, in Lim $18 \mathrm{~mm}$ oder gar $18 \frac{1}{2} \mathrm{~mm}$, wenn man die 3 grössten ( $20 \mathrm{~mm}$ übersteigenden) Stücke noch der kleinen Form zurechnet.

1 Stück $\left(26 \frac{1}{2} \times 20 \times 17\right.$ mm von Fx, Køb. Mus., fig. 9) hat eine besonders hinten monströs verdickte AL., die im letzten Viertel stark linksgebogen und gleichzeitig nach rechts winklig ausgebaucht ist, so dass die Hinterseite des Stk. auffällig breit und flach erscheint. Einzelne andere Stücke sind extrem parallelseitig.

Selten ist die Sp.-Spitze stark vorgezogen, dabei aber nur wenig breiter als bei den gewöhnlichen bullaria mit ganz kleiner, knopfförmiger Sp.-Spitze (fig. 10).

Infolge der grossen Seltenheit genügend erhaltener Schalen oder Abdrücke ist die Zahl der Z. schwerer feststellbar als bei danica; es scheint jedoch, dass bei bullaria eine feinzähnige Form (23-25 AZ., 20 IZ. bei $16-17 \mathrm{~mm}$ und 25 IZ. bei $26 \mathrm{~mm}$ Schalenlänge, in der Zahndichte ungefähr danica entsprechend) von einer grobzähnigen Form (21 AZ., 18 IZ. bei 17-20 mm Länge) zu unterscheiden ist, schliesslich scheinen aber auch besonders feinzähnige Stücke (30 AZ., 28 IZ. bei 18-20 mm Länge) vorzukommen).

An Bruchstücken zeigen die inneren Umgänge eine dichte Spiralrippung ohne Längsstreifung, also die Skulptur der Jugendform von Eocypraea.

\section{Genus Vicetia Fabiani.}

Die ansehnliche Grösse, auffällige Gestalt, breite Sp. und vor allem die Merkmale der Fossula genügen, um in dem unten beschriebenen Unikum mit Sicherheit eine Vicetia zu erkennen; dieses Genus war bisher nur aus dem Eocän bekannt, doch könnte auch schon baluchistanensis Noetl. aus dem Obersenon hierher gehören. 
Vicetia faxensis nov. sp.

Steinkern gestreckt zylindrisch, Sp. sehr breit, wahrscheinlich kurz vorgezogen (beschädigt), M. ziemlich gerade, der vordere Ausguss breit, aber tief; die Schale hatte nach den vorzüglich erhaltenen Abdrücken am Stk. ein nicht ausgebogenes, sehr steil declives VE. der AL. mit tief herabgehenden, kräftigen vorderen AZ.-Falten, im ganzen etwa $32 \mathrm{AZ}$. und eine sehr breite und tiefe, in ihrer ganzen Breite grob quergefaltete Fossula, während die hinteren IZ. auf den M.-Rand beschränkt blieben und nicht auf die Columella fortgesetzt waren; das HE. war wohl wenigstens etwas geschnäbelt. $54 \times 31 \frac{1}{2} \times 24 \mathrm{~mm}$ (Typus von Fx, Køb. Mus., fig. 19).

\section{Genus Palaeocypraea nov.}

Schale spindelförmig, etwas bauchig mit gerundeten S. und spitz ausgezogenen, etwas rückgebogenen E.; Sp. stark vorgezogen, den linken Teil des hinteren M.-Ausgusses bildend, in der Jugend gegittert; M. nur wenig seitlich gelegen, AL. vorn flach decliv, in der Mitte verbreitert, AZ. und IZ. bis zur Spitze des HE. wohl ausgebildet, kurzfaltig, TZ. undeutlich randständig, von kräftigem radialfaltigem Zwischenzahn gefolgt, übrige IZ. quer, Fossula breit, konkav, glatt, mit deutlicher Kerbe am glatten Innenrande. - Typus: Cypraea spirata Schloth.

Das neue Genus umfasst einige fast ausnahmslos mesozoische Arten, die bisher nur gezwungen bei verschiedenen Gattungen untergebracht waren; die Auffindung der so gut erhaltenen Schalen von spirata hat einerseits ihre Zusammengehörigkeit aufgedeckt, andererseits aber erwiesen, dass die ganze Gruppe keinem der bisher aufgestellten Genera angeschlossen werden kann, da sie höchst primitive, sogar zwischen den bisherigen Unterfamilien und Tribus der Cypraeidae vermittelnde Kombination von Merkmalen aufweist. Das Genus Palaeocypraea steht phylogenetisch ${ }^{1}$ ) an der Wurzel der $C y$ -

1) Vergleiche den hypothetischen Stammbaum der Cypraeacea im Arch. Naturgesch., 91, 10, p. 50 (1927) (Schilder). 


\section{18}

praeinae, da von ihm die Bernayini (Protocypraea) und die Erosariini (Conocypraea) in gleicher Weise ableitbar sind, und zeigt auch noch nahe Verwandtschaft mit den primitiven Archicypraeinae: Palaeocypraea sind Protocypraea mit der gegitterten Sp. ${ }^{1}$ ) und den bis zum HE. fortgesetzten Z. der primitiven Erosariini einerseits, mit der lang vorgezogenen, an den hinteren M.-Ausguss angelehnten Sp. von Archicypraea andererseits; von den Conocypraea trennt sie vor allem das Fehlen des aufgebogen gerandeten AR., der stets kräftige Zwischenzahn in der Lücke hinter dem TZ. und die der B. mehr genäherte Sp., von den Archicypraea die grössere Breite der AL., Sp. und Fossula. Auch bei schlecht erhaltenen Arten, die weder Fossula, TZ. noch eine Sp.-Skulptur erkennen lassen, sind die auffällig geschnäbelten, nicht gerandeten E., die mit dem HE. der IL. ganz verschmolzene Sp. und die bis zum HE. deutlichen, dichten Z. stets als Merkmale von Palaeocypraea zu erkennen.

Es gehören demnach hierher:

a) kleinere Arten:

\begin{tabular}{|c|c|c|}
\hline nomala St & Sen. sup. & In \\
\hline lobulina $S$ & Sen. sup. & Indien. \\
\hline quyeri Campb. & Sen. sup. & Montana. \\
\hline Mortoni Gabb & Oberkreid & $\mathrm{N}$ \\
\hline mburgensi & Sen. sup. & Maestricht. \\
\hline
\end{tabular}

1) Die auffällige Gitterskulptur der Sp. war bisher ausser bei den zu den Amphiperasidae gehörenden Gattungen Protocypraedia und Pediculariella nur bei Palaeocypraea squyeri ("strong revolving raised lines and striae «) beschrieben worden; jetzt fand ich sie auch bei jungen Pustularia cicercula Linn. und Nuclearia nucleus Linn. deutlich ausgebildet, nicht mehr aber bei den Erosaria.

$\left.{ }^{2}\right)$ Neuerliche genaue Betrachtung der von Stoliczka gegebenen Abbildung lässt mich meine frühere Annahme, die Sp. sei involut, bezweifeln; die erhaltenen Schalenreste von anomala und globulina ähneln vielmehr sehr spirata, neben die auch Vredenburg beide Arten stellt; Stoliczka vergleicht seine Arten mit Pustularia (»Epona«).

3) Wenigstens das von Wellner abgebildete Stück steht squyeri nahe.

${ }^{4}$ ) Das Køb. Mus. besitzt einen 101/2 $\times 8 \times 6 \frac{1}{2} \mathrm{~mm}$ grossen 
cretacea Orb.

spirata Schloth. libyca Opp.

b) grössere Arten:

maestrichtensis nov. ${ }^{1}$ )

suecica nov.
Dan.

Dan.

Lib. inf.

Sen. sup.

Dan.
Beynes, Vigny

(Oise)

Dänemark, Skåne.

Aegypten.

\section{Palaeocypraea spirata Schlotheim.}

= Cypraeacites spiratus Schlotheim (1820) Petrefakt., p. 1182). = Cypraea spirata Ravn (1902) Kong. Danske Vidensk. Selsk. Skr., Mat. Mat. Afd., (6) 11, tab. 2, fig. 2 (nec fig. 1 nec fig. 3).

Schale bauchig elliptisch mit spitz ausgezogenen, etwas rückgebogenen E., S. gerundet, auch an den E. nicht gerandet, Sp. bei adulten Stücken unter Callus verdeckt und in ihrer Lage in der linken Hälfte des HE. kaum erkennbar; M. hinten etwas gebogen, AL. vorn deutlich decliv, AZ. hier faltenförmig verlängert (die vordersten wieder kürzer), TZ. undeutlich randständig, mit schwachem Knopf beim Eintritt in das Innere der M., der nachfolgende Zwischenzahn schrägfaltig, die vorderen IZ. quer, knopfförmig, die hinteren kurz querfaltig, die 3 letzten auf dem die Sp. bedeckenden Callus selbst aufsitzend, auch die hinteren, etwas verlängerten $\mathrm{AZ}$. reichen bis zur Schalenspitze; Fossula ziemlich breit, seicht, glatt, der Innenrand bildet hinter dem den Vorderrand bildenden, vom

Stk. von Valkenburg (Maestricht), der von spirata durch die viel gröberen $Z$. (nach dem Abdrucke nur 16-18 AZ.) gut zu unterscheiden ist; die Gestalt des Stk. ist vorn bauchig verschmälert, also spirata näherkommend als suecica, die Sp. vielleicht etwas weniger vorspringend, aber sonst wie bei spirata gebildet.

1) Diesen Namen schlage ich für die von mir bereits im Arch. Naturgesch. 91, 10, p. 89, 138, Anm. 185 (1927) abgetrennte grössere Form von Maestricht vor (Kaunhowen (1898) Paläont. Abhandl., 8, 1, tab. 9, fig. 1), die suecica nahezustehen scheint.

$\left.{ }^{2}\right)$ Schlotheim's Angaben über die sehr vortretende Sp. und die geringe Grösse des Stk. ("weniger als $1 / 2$ Zoll lang«) beweisen, dass ihm weder globuliformis noch suecica vorgelegen haben. 
TZ. ausgehenden Kiel eine deutliche Kerbe und ist dahinter wieder etwas ausgebogen und schwach angeschwollen. 131/2 $\times$ $9 \times 7 \frac{1}{2} \mathrm{~mm}$ mit $20 \mathrm{AZ}$. und $17 \mathrm{IZ}$. (Exemplar von FM, Køb. Mus., fig. 3).

Etwas jüngere Schalen haben die Sp.-Umgänge deutlicher sichtbar, VE. der AL. weniger decliv, Z. kürzer. 141/2 $\times 9 \frac{1}{2} \times$ $8 \mathrm{~mm}$ mit $22 \mathrm{AZ}$. und $19 \mathrm{IZ}$. (Exemplar von FM., Køb. Mus., fig. 4).

Noch jüngere Schalen zeigen an der Sp. (selten auch auf der IL.) eine deutliche Gitterskulptur, bestehend aus alternierend stärkeren und schwächeren Spiralrippen, gekreuzt von Längsleistchen ${ }^{1}$; die IZ. sind bis zur Mitte des drittletzten Umganges auf einem callösen Kiel knopfförmig fortgesetzt; VE. der AL. wenig decliv, alle Z. kurz, z. T. noch undeutlich. VE. weniger vorgezogen, das $\mathrm{HE}$. der AL. verbindet sich dünn mit der Sp.-Spitze(!), ohne dass ein deutlicher hinterer Ausguss der M. entsteht (vgl. Archicypraea). $13 \frac{1}{2} \times 9 \times 7 \mathrm{~mm}$ mit $21 \mathrm{AZ}$. und $18 \mathrm{IZ}$. (Exemplar von FM., Køb. Mus., fig. 5).

Ganz junge Schale: Sp. gegittert, übrige Sch. längsgestreift, AL. noch scharfrandig, ohne Z. (Bruchstück von FM., Køb. Mus.).

Steinkern kurz zylindrisch, E. gerundet, Sp. breit, mit allen Umgängen (auch dem Protoconch!) stark vorspringend, von der Ausfüllung des hinteren M.-Ausgusses rechts nur zum Teil bedeckt. $11 \times 10 \times 8 \mathrm{~mm}$ mit Abdrücken von 19 AZ. und 16 IZ. (Neotypus ${ }^{2}$ ) von Fx., Køb. Mus., fig. 12; die fig. 13 zeigt die Lage eines $12 \times 10 \frac{1}{2} \mathrm{~mm}$ grossen Stk. in den wohlerhaltenen Umrissen der dazugehörigen Schale von $17 \frac{1}{2} \times 103 / 4 \mathrm{~mm}$ ).

Abänderungen: Nachstehend wieder eine Uebersicht der beobachteten Stk.-Längen:

1) Vgl. auch die Abbildung bei Ravn! - Diese Gitterskulptur ist auch auf einem Steinkern von Ag., allerdings kaum mehr erkennbar, abgedrückt.

2) Vgl. Fussnote 1 auf p. 15 . 
3. 21

\begin{tabular}{c|c|c|c|c|c|r|r|r}
\hline \multirow{2}{*}{$\mathrm{mm}$} & \multicolumn{7}{|c}{ Ort } \\
\cline { 2 - 9 } & $\begin{array}{c}\text { Fx } \\
\text { (normal) }\end{array}$ & $\begin{array}{c}\text { Fx } \\
\text { (kugelig) }\end{array}$ & St & FM & Ag & Lim & Summe & oder \\
\hline 6 & - & - & - & - & - & 1 & 1 & 1 \\
7 & - & - & - & - & - & - & - & 4 \\
8 & - & - & - & - & 1 & 3 & 4 & 4 \\
9 & 5 & 1 & - & 2 & 4 & 7 & 19 & 67 \\
10 & 22 & 2 & - & 6 & 4 & 14 & 48 & 67 \\
11 & 49 & 4 & - & 6 & 4 & 9 & 72 & 180 \\
12 & 81 & 4 & - & 4 & 3 & 16 & 108 & \\
13 & 53 & 6 & - & 2 & 3 & 5 & 69 & 101 \\
14 & 24 & 1 & - & - & 3 & 4 & 32 & \\
15 & 11 & 1 & 1 & 1 & 1 & - & 15 & 24 \\
16 & 7 & 1 & - & - & - & 1 & 9 & \\
17 & 2 & - & - & - & - & - & 2 & 2 \\
\hline Mittel in & 12.3 & 12.2 & $?$ & 11.1 & 11.3 & 11.0 & & \\
mm & & & & & & & & \\
\hline
\end{tabular}

Man sieht, dass zu der im Mittel über $12 \mathrm{~mm}$ langen Form von Fx und St an den übrigen Fundorten (FM, Ag, Lim) noch eine kleinere, etwa $10 \mathrm{~mm}$ lange Form hinzutritt, deren Existenz durch die zweigipfelige Variationskurve von Lim besonders in Erscheinung tritt und die das Mittel der Gesamtpopulation dieser Fundorte auf etwa $11 \mathrm{~mm}$ herabdrückt.

Die mehr kugelige, das heisst breitere Varietät mit mehr gerundeten S. (fig. 14) ist von der typischen mehr parallelseitigen Form in der Grösse nicht verschieden und geht auch in der Gestalt in sie allmählich über. Die 4 grössten Stücke von Ag sind vorn ein wenig mehr gleichmässig verschmälert, ähneln also etwas einer extrem kleinen suecica; die Stücke sind aber zu sehr beschädigt, um ein endguiltiges Urteil über sie abgeben zu können.

Die Zahl der Zähne beträgt bei der

Sch.-Länge $13-15 \mathrm{~mm}=$ Stk.-Länge $9-10 \mathrm{~mm}$ meist $20-23 \mathrm{AZ}$. und $17-20 \mathrm{IZ}$,

Sch.-Länge $16-17 \mathrm{~mm}=$ Stk.-Länge $11-12 \mathrm{~mm}$ meist 20-23 AZ. und 18-21 IZ.,

Sch.-Länge $19 \mathrm{~mm}=$ Stk.-Länge $13 \mathrm{~mm}$ meist 24 AZ. und $23 \mathrm{IZ}$. 


\section{Palaeocypraea suecica nov. sp.}

= Cypraea spirata Ravn (1902) Kong. Danske Vidensk. Selsk. Skr., Nat. Mat. Afd., (6) 11, tab. 2, fig. 1, 3 (nec fig. 2, nec Schlotheim).

Steinkern wie bei spirata, aber stets bedeutend grösser ${ }^{1}$ ), der AR. von $2 / 3$ der Länge an nach vorn gleichmässig leicht gebogen, die Sp. breiter und oft noch stärker vorgezogen. $30 \times 22 \times 18 \mathrm{~mm}$ (Typus von Lim, Køb. Mus., fig. 17; ein besser erhaltenes Stück, $31 \times 22 \times 18 \mathrm{~mm}$, von Fx, Køb. Mus. ist in fig. 18 abgebildet).

Stücke mit erhaltener Schale sind mir unbekannt; das VE. der Sch. muss jedoch sehr stark vorgezogen gewesen sein (fig. 16).

Abänderungen: Die Variabilität in der Grösse ist bei suecica relativ geringer als bei spirata:

\begin{tabular}{c|r|r|r|r}
\hline \multirow{2}{*}{$\mathrm{mm}$} & \multicolumn{4}{|c}{ Ort } \\
\cline { 2 - 5 } & Fx & Lim & Summe & oder \\
\hline 17 & - & 1 & 1 & \\
18 & 1 & 2 & 3 & 4 \\
19 & 1 & 4 & 5 & 15 \\
20 & 4 & 6 & 10 & \\
21 & - & 4 & 4 & 10 \\
22 & 1 & 5 & 6 & \\
23 & 5 & 6 & 11 & 23 \\
24 & 5 & 7 & 12 & \\
25 & 6 & 9 & 15 & 29 \\
26 & 5 & 9 & 14 & \\
27 & 1 & 5 & 6 & 12 \\
28 & 1 & 5 & 6 & \\
29 & 1 & 1 & 2 & 3 \\
30 & 1 & - & 1 & \\
31 & 2 & 2 & 4 & 5 \\
32 & - & 1 & 1 & \\
\hline Mittel in & 24.4 & 24.0 & & \\
mm & & & \\
\hline
\end{tabular}

$\left.{ }^{1}\right)$ Die Variationsbreiten von spirata und suecica überkreuzen einander praktisch gar nicht, die Variationskurve der Summe aller 
Man kann erkennen, dass eine kleine (20 mm), eine mittlere $(25 \mathrm{~mm})$ und eine grosse Form $(31 \mathrm{~mm})$ als Kurvengipfel der Variationsreihe zu unterscheiden sind, und zwar an beiden Fundorten, die im übrigen in der Grösse der Steinkerne fast vollständig übereinstimmen.

Ein Stk. von $20 \mathrm{~mm}$ Länge zeigt Abdrücke von etwa 28 AZ., ein anderer von $28 \mathrm{~mm}$ Länge anscheinend $29 \mathrm{IZ}$.

\section{Genus Protocypraea Schilder.}

Schon die Abbildung RAVN's liess mich ${ }^{1}$ ) in globuliformis eine Protocypraea erkennen; das Genus kommt zwar mit 1 Art sogar rezent vor, die Hauptmasse der hierhergehörenden Arten ist aber mesozoisch; globuliformis kann ebenso mit gemmellaroi di Stef. aus dem Tithon von Sizilien wie mit Schlotheimi Schild. aus dem Mitteleocän vom Kressenberg verglichen werden.

\section{Protocypraea globuliformis Ravn.}

= Cypraea globuliformis Ravn (1902) Kong. Danske Vidensk. Selsk. Skr., Nat. Mat. Afd., (6) 11, p. 227, tab. 2, fig. 6 a-d.

Steinkern kugelig, mit breiter, nur wenig vorgezogener Sp., deren Spitze nur selten aus dem niedrigen Kegel der übrigen Umgänge vorragt und dann (im Gegensatze zu Palaeocypraea spirata) fein und schmal ist; M. hinten gebogen, AL. konvex ohne besonders scharfen Kiel, IL. in ${ }^{1 / 3}$ der Länge an M. schwach eingedrückt. $11 \frac{1 / 2}{2} \times 9 \frac{1}{2} \times 8 \frac{1}{2} \mathrm{~mm}$ (Typus von Fx, Køb. Mus., von Ravn abgebildet; ein besser erhaltenes Stück, $11 \times 10 \frac{1}{2} \times 9 \frac{1}{2} \mathrm{~mm}$ von ebenda zeigt fig. 15).

Nach den vorhandenen Abdrücken war die Schale wahrscheinlich etwas geschnäbelt, die AZ. fein wie bei Palaeo-

Stücke beider Arten ist deutlich zweigipfelig, auch bei Zusammenziehung von je $3 \mathrm{~mm}$ (5-7, 8-10 mm usw.): 1, 71, 249, 56, 11, $20,38,26,7,1$. An der Artberechtigung von suecica wäre demnach auch ohne den auffälligen Unterschied in der Verschmälerung des VE. und die verschiedene Verbreitung nicht zu zweifeln.

1) Schilder (1927) Arch. Naturgesch., 91, 10, p. 89. 
cypraea spirata (Zahl nicht sicher angebbar!), die Foss. breit, etwas konkav, mit verstärktem Vorderrande, aber anscheinend ohne eine Kerbe am Innenrande und nur aussen von den vordenen IZ. betreten, also im übrigen glatt und glattrandig.

Abänderungen der auf $\mathrm{Fx}$ beschränkten Art sind auch in der Grösse nicht bedeutend:

\begin{tabular}{c|c|c|c|c|c|c}
\hline Ort & 10 & 11 & 12 & 13 & $14 \mathrm{~mm}$ & Mittel in mm \\
\hline Fx & 1 & 2 & 5 & 5 & 3 & 12.6 \\
\hline
\end{tabular}

\section{ERGEBNISSE.}

1. - Zwischen der Cypraeacea-Fauna der 5 Fundorte des oberen Danium besteht eine weitgehende Uebereinstimmung betreffs der sie zusammensetzenden Genera, und zwar sowohl bezüglich der überhaupt vorkommenden Genera als auch bezüglich ihrer relativen Häufigkeit.

\begin{tabular}{r|r|r|r|r|c}
\hline Ort.... & St & Fx & Ag & FM & Lim \\
\cline { 2 - 6 } Stücke. & 1 & 500 & 32 & 39 & 208 \\
\hline davon \% Cypraedia ..... & - & 5 & 6 & 10 & 10 \\
Eocypraea ..... & - & 30 & 22 & 36 & 29 \\
Vicetia ...... & - & 0.2 & - & - & - \\
Palaeocypraea & 100 & 62 & 72 & 54 & 61 \\
Protocypraea .. & - & 3 & - & - & - \\
\hline
\end{tabular}

Palaeocypraea bildet also überall rund zwei Drittel, Eocypraea ein Viertel, Cypraedia ein Fünfzehntel der Cypraeacea-Fauna; die alleinige Auffindung des Unikum von Vicetia an dem individuenreichsten Fundorte besagt nichts, wohl aber ist das Fehlen von Protocypraea an allen Fundorten ausserhalb Fx auffällig.

2. - Dagegen bestehen zwischen den einzelnen Fundorten wesentliche Unterschiede in der Häufigkeit der zu den Genera gehörenden Arten: 


\begin{tabular}{|c|c|c|c|c|c|c|c|}
\hline \multirow{2}{*}{ Eocypraea } & Ort.... & \multicolumn{2}{|l|}{$\mathrm{Fx}$} & $\mathrm{Ag}$ & \multicolumn{2}{|c|}{ FM } & $\operatorname{Lim}$ \\
\hline & Stücke.. & \multicolumn{2}{|c|}{151} & 7 & \multicolumn{2}{|c|}{14} & 61 \\
\hline \multicolumn{2}{|c|}{$\begin{array}{r}\text { davon } \% \text { danica } \ldots \ldots \ldots . \\
\text { bullaria } \ldots \ldots \ldots\end{array}$} & \multicolumn{2}{|l|}{$\begin{array}{l}27 \\
73\end{array}$} & $\begin{array}{l}14 \\
86\end{array}$ & \multicolumn{2}{|c|}{$\begin{array}{l}79 \\
21\end{array}$} & $\begin{array}{l}59 \\
41\end{array}$ \\
\hline \multirow{2}{*}{ Palaeocypr } & Ort..... & St & $\mathrm{Fx}$ & \multicolumn{2}{|c|}{$\mathrm{Ag}$} & $\mathrm{FM}$ & $\mathrm{Lim}$ \\
\hline & Stücke. . & 1 & 308 & \multicolumn{2}{|c|}{23} & 21 & 127 \\
\hline davon $\% s$ & $\begin{array}{l}\ldots \ldots \\
\ldots \ldots\end{array}$ & $\underline{100}$ & $\begin{array}{l}89 \\
11\end{array}$ & \multicolumn{2}{|c|}{$\begin{array}{r}100(83 ?) \\
-(17 ?)\end{array}$} & $\underline{100}$ & $\begin{array}{l}47 \\
53\end{array}$ \\
\hline
\end{tabular}

In Lim sind die beiden Arten in jedem Genus etwa gleich häufig, während an den übrigen Fundorten jeweils 1 Art überwiegt; bei Eocypraea stehen Fx + Ag im Gegensatze zu FM, Lim ist gewissermassen intermediär; auch bei Palaeocypraea ist der gleiche Gegensatz von $\mathrm{Fx}+\mathrm{Ag}$ zu FM erkennbar, wenn man die 4 grössten, leider schlecht erhaltenen spirata von Ag zu suecica rechnet.

3. - Weitere Unterschiede zwischen den einzelnen Fundorten ergeben sich bei Betrachtung der Verbreitung der Unterarten und Abänderungen nach der Grösse:

a) Cypraedia

\begin{tabular}{c|c|c|c|c}
\hline Ort.... & Fx & Ag & FM & Lim \\
\hline Stücke.. & 24 & 2 & 4 & 20 \\
\hline$\ldots \ldots \ldots \ldots$ & - & 100 & - & - \\
$\ldots$ Form).. & 58 & - & - & 50 \\
$\ldots \ldots \ldots . .$. & 38 & - & 25 & 30 \\
$\ldots$ & 4 & - & 75 & 20 \\
\hline
\end{tabular}

b) Bei Eocypraea danica ist die kleinere Form in Fx (und $\mathrm{Ag}$ ?) häufiger als in FM und Lim.

c) Bei Eocypraea bullaria bildet die grössere Form in Fx $35 \%$, sonst fehlt sie ganz.

d) Bei Palaeocypraea spirata, wo die kleinere und grössere 
Form schwer trennbar und jedenfalls nicht in \% anzugeben sind, ist die kleinere in $\mathrm{Fx}$ (und $\mathrm{St}$ ?) jedenfalls viel seltener als an den übrigen Orten.

e) Bei Palaeocypraea suecica scheinen die kleine (18 bezw. $25 \%$ ), mittlere (73 bezw. $71 \%$ ) und grosse Form (9 bezw. $4 \%$ ) in Fx (34 Stück) und Lim (67 Stück) ziemlich gleich häufig zu sein.

4. - Eine gute Uebersicht über die Beziehungen der einzelnen Fundorte bietet auch die nachstehende Tabelle, auf der mit 1 jeweils derjenige Fundort bezeichnet ist, wo die betreffende Art am kleinsten, mit 4 derjenige, wo sie am grössten ist:

\begin{tabular}{|c|c|c|c|c|}
\hline Ort..... & $\mathrm{Fx}$ & $\mathrm{Ag}$ & FM & $\operatorname{Lim}$ \\
\hline Cypraedia separabilis ..... & 2 & 1 & 4 & 3 \\
\hline Eocypraea danica ........ & 1 & 2 & 3 & 4 \\
\hline bullaria ........ & 2 & 1 & 3 & 4 \\
\hline Palaeocypraea spirata..... & 4 & 3 & 2 & 1 \\
\hline suecica .... & 4 & - & - & 1 \\
\hline
\end{tabular}

Man erkennt ohne weiteres

a) die Verwandtschaft der Cypraeacea-Fauna von Fx mit $\mathrm{Ag}$ (sowie St) einerseits, von FM mit Lim andererseits;

b) einen grundsätzlichen Unterschied in der Grössenentwicklung zwischen den zwei vertretenen Familien an diesen beiden Fundortgruppen, indem die Amphiperasidae in den stratigraphisch jüngeren Fundorten FM und Lim grösser, die Cypraeidae dagegen kleiner werden.

5. - Bei Untersuchung der Frage, ob die Cypraeacea des Danium von Dänemark und Schonen mehr mesozoischen oder tertiären Charakter haben, darf nicht vergessen werden, dass wir die eocäne Fauna Europas viel besser kennen als die cretacische; das Danium von Faxe und Limhamn stellt die älteste der an Arten und Individuen reichen Faunen Europas dar, die einzige noch ältere reiche Fauna der Erde ist das Senon von Südindien, das aber z. T. ganz anders geartete Cypraeacea enthält. 
Cypraedia und Vicetia sind zwar bis jetzt nur aus dem Tertiär bekannt, doch ist ihre Existenz auch schon in der Oberkreide sehr wahrscheinlich; Eocypraea lebt vom Albian bis ins Neogen, ist also für die Entscheidung der Frage ohne Bedeutung, obwohl manche Tatsachen dafür sprechen, dass die Hauptentfaltung der echten Eocypraea in der Kreide lag; Palaeocypraea ist dagegen ein ausgesprochen mesozoisches Genus mit sehr nahe verwandten Formen im Obersenon, und auch die Protocypraea haben unzweifelhaft in der Oberkreide ihre Blüte gehabt.

Die Cypraeacea-Fauna des Danium spricht also eher für seine Zuteilung zur Kreide als zum Tertiär. 


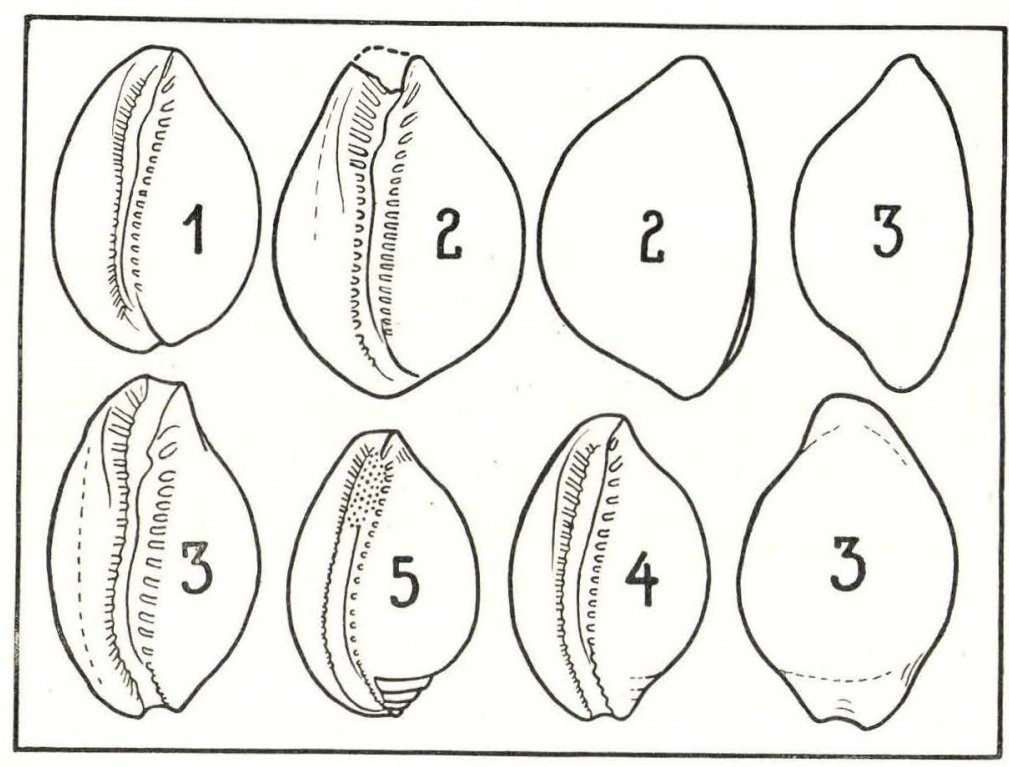

1, 2, Eocypraea danica Schilder. - 3, 4, 5, Palaeocypraea spirata Schloth. (Näheres siehe im Text.)

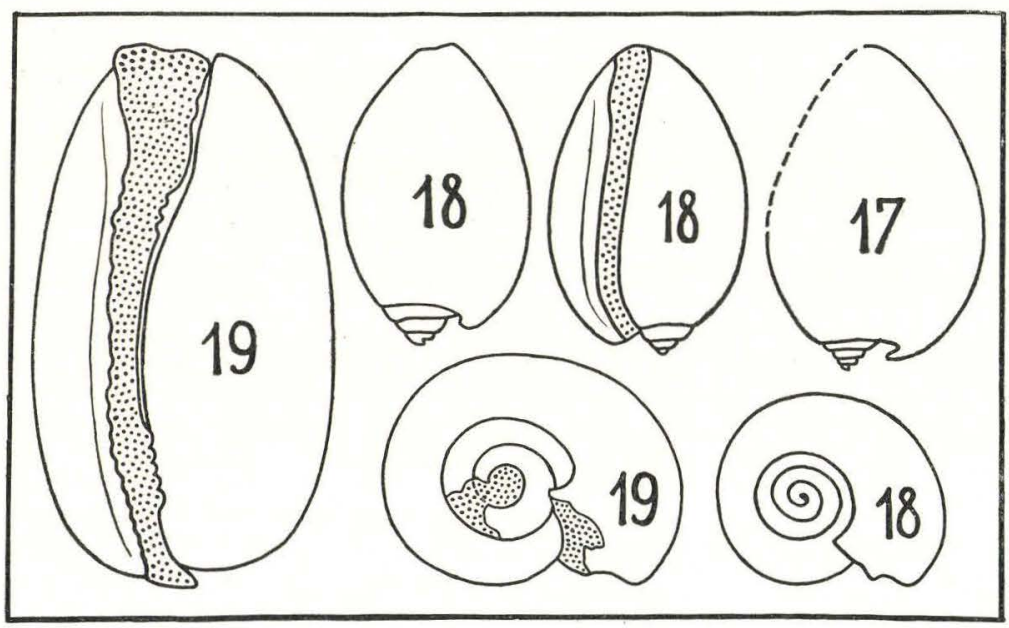

17, 18, Palaeocypraea suecica nov. sp. - 19, Vicetia faxensis nov. sp. (Näheres siehe im Text.) 


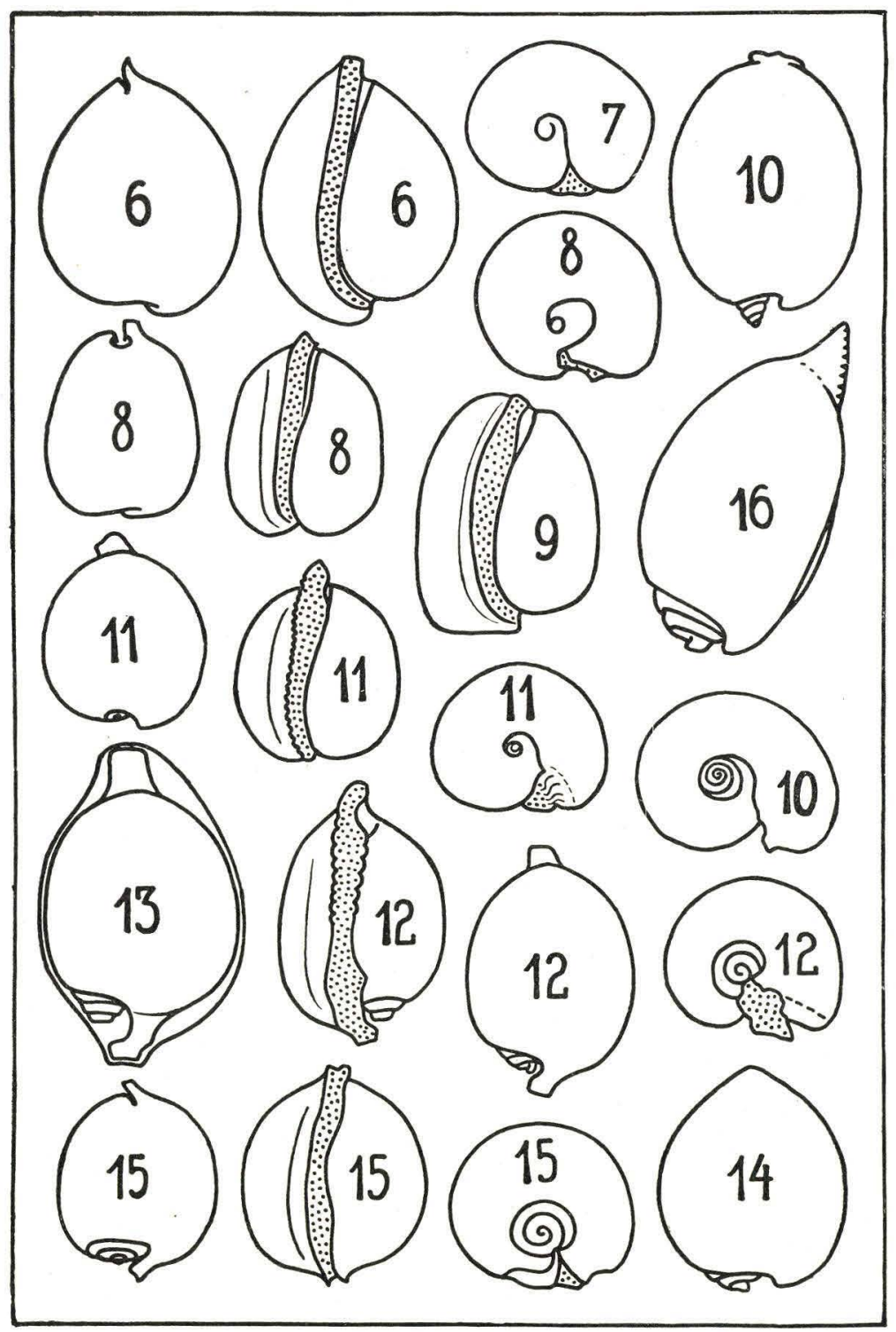

6, Cypraedia separabilis nov. sp. - 7, Cypraedia Ravni nov. sp. - 8, 9, 10, Eocypraea bullaria Schloth. - 11, Eocypraea danica Schilder. - 12, 13, 14, Palaeocypraea spirata Schloth. - 15, Protocypraea globuliformis Ravn. 16, Palaeocypraea suecica nov. sp. (Näheres siehe im Text.) 
